\title{
Development of Intervention Package among Main Care-givers of Pregnant Women and Its Effect on Continuum of Care: Study Protocol for Quasi- experimental Study in Rural Areas of Yangon Region, Myanmar
}

\author{
May Soe Aung ${ }^{1}$, Myint Moh Soe ${ }^{2}$, Yee Myint Swe ${ }^{3}$ \\ ${ }^{1}$ Department of Preventive and Social Medicine, University of Medicine, Yangon, Myanmar; \\ ${ }^{2}$ Maternal and Reproductive Health Division, Department of Public Health, Ministry of Health and Sports, Nay Pyi Taw, \\ Myanmar; \\ ${ }^{3}$ Department of Population and Family Health, University of Public Health, Yangon, Myanmar
}

DOI: 10.29322/IJSRP.10.03.2020.p9934

http://dx.doi.org/10.29322/IJSRP.10.03.2020.p9934

\begin{abstract}
Evidence-based findings of completion of continuum of care among pregnant women can be proved to fulfill further activities for improving maternal health. It is aimed to develop intervention package for main care givers of pregnant women and to study its effect on continuum of care. Quasi-experimental study is done among 200 pregnant women and their main care givers from rural areas of Yangon region. To develop intervention package, Focus Group Discussions of pregnant women, main-care givers and midwives is done. Continuum of care among pregnant women and awareness of main care givers will be assessed by pretested questionnaire in both intervention and control cluster. Perceptions on continuum of care among main-care givers will be explored by In-depth Interview. Three Focus Group Discussions was conducted including pregnant women, main care-givers and midwives in separate session respectively. This qualitative analysis is ongoing for development of intervention package. Pretesting for quantitative data collection was done among 20 pregnant women and their main care-givers. The health education intervention for main care- givers of pregnant women will be done by trained midwives. Outcomes assessment are expected to be completed by June 2021 by mixed methods. By developing health education package for main care givers, important factors for health of pregnant women can be reached to family level within community and the results are hope to be helpful for current activities of maternal health promotion in Myanmar.
\end{abstract}

Index Term: Intervention Package, Main Care-givers, Pregnant Women, Continuum of Care, Myanmar

\section{I.INTRODUCTION}

Maternal health from pregnancy to post-natal period is inseparable for the needs for health care. The links between the home and the first level health care facility and improving health status of mothers can be strengthened by an effective continuum of care $(\mathrm{CoC})[1,2]$. An operational continuum of care with accessible, high quality care is essential before and during pregnancy, childbirth, and the postnatal period for preventing problems for mothers and babies. The dimensions of continuum of care by WHO guide to address continuous care from home to health center and hospital [3]. A systematic review suggested that continuous uptake of antenatal care, skilled birth attendance, and postnatal care is necessary to improve maternal health outcomes 
in low- and middle-income countries [4].The WHO ANC model highlighted to have first ANC contact during the first 12 weeks' gestation, followed by contacts at 20,26, 30, 34, 36, 38 and 40 weeks' gestation. The number of ANC visits is recommended from four to a minimum of eight times [5]. Pregnancy related complication can be appropriately managed by the care of a health professional with midwifery skills because of early recognition of danger signs and an effective timely referral to a well-equipped facility [6]. The postnatal period following childbirth is a vulnerable phase in the lives of mothers [7]. Therefore, the WHO recommended mothers to receive postnatal care within the first 24 hours followed by postnatal check-up on the second or third day, and then on the seventh day after delivery [8]. Improved coverage of ANC, delivery attended by SBA and PNC as continuum of care among pregnant women have been regarded as keys to improve maternal health outcomes [1].

In Myanmar, MMR was 250 per100,000 live births in 2017 which is higher compared to that of South East Asia region ,137 per 100.000 live births [9]. Completing continuum of care among Myanmar pregnant women is important to reduce MMR to 91 per 100,000 live births by 2030 [10, 11, 12]. However, there are some problems to fulfill continuum of care of pregnant women in Myanmar because of inadequate antenatal care, unawareness on importance of early antenatal visit, antenatal timing and minimum antenatal care frequency, unrecognition of 6 weeks post-natal periods and incomplete post-natal care [13,14,15]. Myanmar women from rural areas are not utilizing the ANC services adequately [16]. The ANC and delivery by skilled birth attendants, number of ANC visit in third trimester of pregnancy, receiving complete health education package were significantly associated with PNC utilization among mothers in hard to reach areas of Myanmar [17].Antenatal care booking and post-natal utilization are related by social factors such as influenced of mother-in-law, relatives' advices, male involvement and misconceptions [18,19]. Therefore, the WHO recommended to involve men and mobilize community during pregnancy, childbirth and after birth, particularly in rural settings with low access to health services [20,21]. For maternal health improvement, the WHO also proposed interventions at the individuals, families and community level where the role of men and other influential of family are mentioned important for awareness raising. For interpersonal processes, social support and working together within families, health education intervention is highlighted as a broad perspective [22].

Evidence-based findings of completion of $\mathrm{CoC}$ among pregnant women can be proved to fulfill further activities for improving maternal health. However, there is still limited findings of interventions regarding CoC in research areas of Myanmar. Especially in the area of improving family and main care giver's role for promoting maternal health, intervention-based findings are limited and needed to be strengthened by conducting further researches. This study intends to develop new intervention package to provide health messages for main care givers of pregnant women and to study its effect on maternal health outcomes by receiving continuum of care. The results of the study is hoped to be useful in taking CoC among pregnant women by identifying role of main care-givers within the community.

\subsection{Study design}

\section{II.METHODOLOGY}

This is a community-based exploratory sequential mixed method design for two years in Taik Kyi township and Kyauktan township of Yangon region as intervention and control cluster. Qualitative data collection by Focus Group Discussions (FGD) among pregnant women, main-care givers and midwives was done in the intervention township to develop intervention package for main care-givers of pregnant women. Final outcomes of the research will be assessed by quantitative data collection in both clusters and In-depth Interview of main-care givers from intervention cluster.

\subsection{Study population}

For FGD, inclusion criteria are pregnant women with any gestational periods; main care- givers of any sex with the age between 18 years and 65 years and currently living with pregnant women under the same shelter; and midwives who are currently working at rural health centers (RHC) and subcenters. For In-depth Interview, criteria for main care-givers of pregnant women is similar to that of FGD. For quantitative study, pregnant women aged 18 years and above; and with pregnancy of not later than 20 
weeks gestation are eligible. Inclusion criteria for main care-givers are husband or other person who are most available to give continuous care at any time for pregnant women; can give pregnancy related decision; living together with pregnant women under same shelter up to 6 weeks after delivery; and any sex with the age between 18 years and 65 years.

\subsection{Sample size determination}

Total minimum required sample is 200 including 100 pregnant women and their main care-givers for each group. The sample size is calculated with proportion of four and more ANC visits in control group, 0.59 [23], and assumed proportion in intervention group as 0.9. Alpha error is considered as 0.05 with $80 \%$ power, $10 \%$ attrition rate and design effect 2.

\subsection{Sampling Procedure}
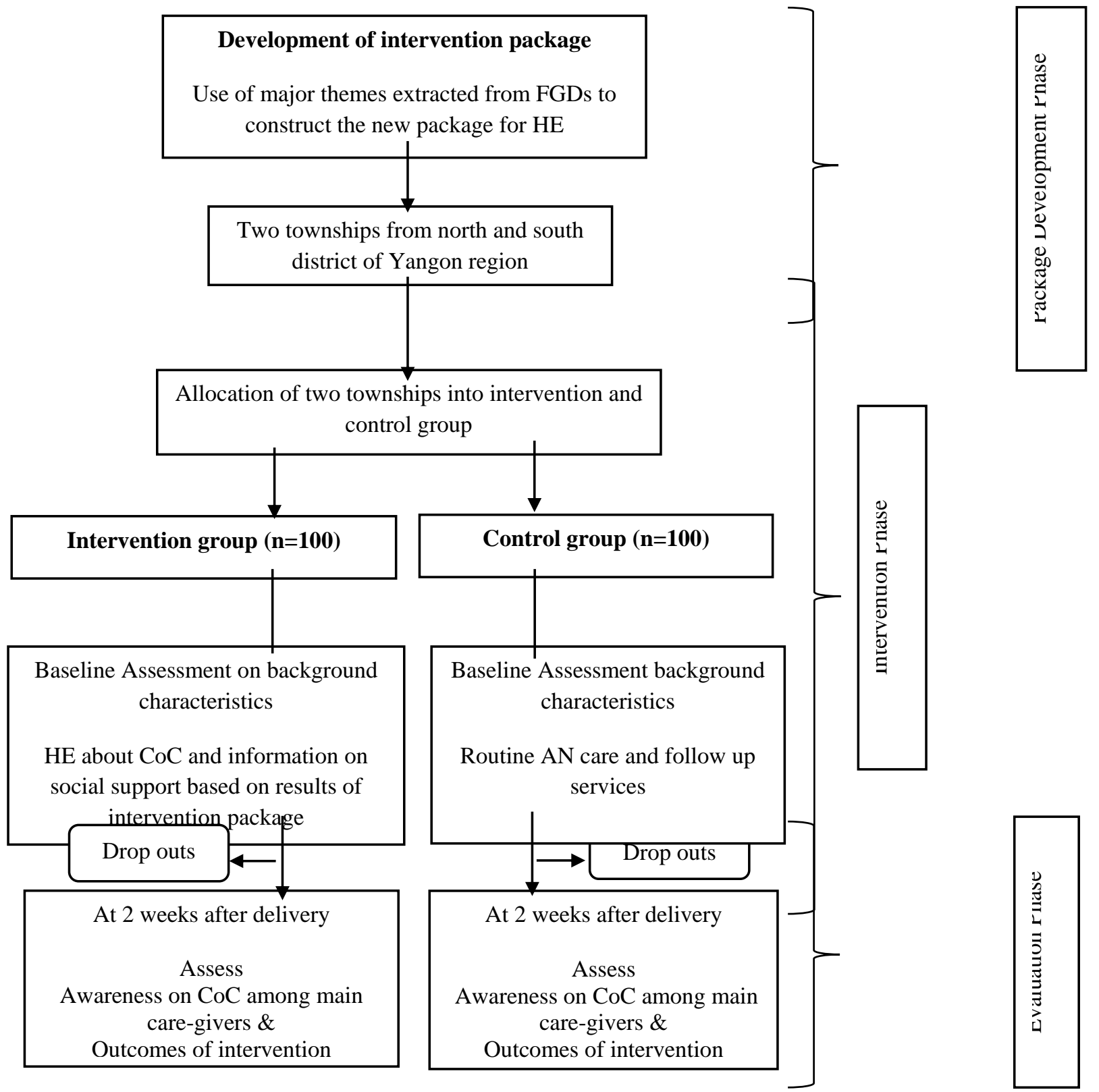

Figure 1: Study flow chart of intervention package development and sampling procedures

Figure 1 summarizes the study flow and sampling procedures. For qualitative method for intervention package development, purposive non probability sampling method is used and all pregnant women, their main care-givers; and all midwives of study area are invited by the help of local authority to participate in FGD. For quantitative method, firstly, north and south districts of Yangon region are purposely chosen for prevention of contamination. Kyauktan township and Taik Kyi where there is adequate rural setting and different classes of people are residing were selected among 8 and 10 townships in north and 
south districts respectively. In both townships, there is more than 3000 pregnant women and rate of AN care for four and more times is less than $85 \%$. From each cluster, two to three RHC villages are selected and all pregnant women and their main caregivers who meet inclusion criteria will be chosen through a consecutive sampling for calculated sample size. For end-line qualitative method, to explore the in-depth ideas of main care givers on continuum of care, In Depth Interview (IDI) will be done among purposively chosen 5 main care-givers of pregnant women who completed $\mathrm{CoC}$ and 5 main care-givers of pregnant women who does not complete CoC respectively. At least 8 antenatal visits, birth by skilled attendance and PNC within 24 hours after birth followed by two additional checks will be considered as criteria of CoC completion. No fulfillment of any one of these three criteria will be taken as incomplete CoC.

\subsection{Data collection methods and tools}

\section{First Phase: Qualitative data collection for intervention package development}

FGDs was conducted by guideline with 7 to 9 participants per session. Before data collection, objectives will be explained and written consent; and permission to record their insights are obtained. All FGDs are held at places with privacy without environmental disturbances. The researcher act as a moderator and note taking is be done by two trained note-takers. Before conclusion, information obtained are confirmed with the participants to ensure what they meant in the discussion.

\section{Second Phase: Quantitative data collection}

Pretesting was carried out among 20 pregnant women and their main care-givers residing apart from study areas. Questionnaire is prepared based on Myanmar MCH Hand Book [24] and Myanmar DHS (2015-16) [23]. Face to face interview will be applied for quantitative data collection. The survey questions comprised of socio-demographic factors of pregnant women and main care-givers and background obstetrics characteristics of pregnant women. Questionnaire on awareness of CoC is used to assess main care-givers from both clusters regarding ANC, danger signs, safe delivery and PNC. Questionnaire for assessing the outcome of intervention among pregnant women included continuum of care by ANC, safe delivery and PNC.

\section{Recruitment and training of midwives}

Midwives from two to three RHCs of both clusters will be recruited on the last day of a month in their respective township. The investigator will explain the trial procedures and their roles in the study. The midwives from intervention group will be trained by principal investigator and requested not to share the information got from training sessions to others apart from the participants in the intervention group up to the intervention period. In the training of midwives, the researcher will use the pamphlet in Myanmar language based on the finding of intervention package and MCH handbook [24] to give health education for main care-giver of pregnant women. The content of pamphlet will include importance of AN visit, care during pregnancy, safe delivery as first portion and danger signs during pregnancy, at birth and after delivery as the second portion.

\section{Baseline data collection}

In both intervention and control groups, baseline data will be collected at first AN visit of eligible pregnant women by midwives. For pregnant women who come alone for AN visit, main care-giver will be invited to be along with her at second AN visit.

\section{Intervention package for intervention group}

Intervention procedures will be done two weeks after the baseline data collection. Main care-givers of pregnant women will be educated by trained midwives about all component of health education pamphlet provided by principle investigator. Health education will take about 30 minutes after routine AN care procedure. After health education session, floor is opened for discussion and individual session will be given if it is requested. Trained midwives from intervention group will be monitored by a checklist and conducting health education will be confirmed by asking the main care-givers.

\section{Comparison group}


Timing and procedures of recruitment is similar to that of intervention group. All eligible pregnant women will be given routine AN care and follow-up care by midwives.

\section{Third Phase: Awareness and outcome assessment}

Awareness on continuum of care among main care-givers and completion of continuum of care among pregnant women in both groups will be assessed after 2 weeks puerperium period. The principle investigators and trained research assistants will collect end line quantitative data by face to face interview using pre-tested questionnaire.

\section{End line qualitative data collection}

After outcome assessment among pregnant women, In Depth Interview (IDI) will be done by principle investigator to explore perception of main care giver on continuum of care. Before conducting data collection, objectives will be explained and written informed consent will be obtained. All places for IDI will be considered for privacy and recordings will be done with permission. Information obtained from IDI will be confirmed with each and every participant to be ascertained what they mean in the interview.

\subsection{Data management and analysis}

\section{First Phase: Qualitative data analysis for exploratory design by FGDs}

The interviewed data will be analyzed manually and the interviews will be transcribed on the day of data collection. Reading and checking of the transcripts by listening of audio records will be done repeatedly by the researcher. Then data will be sorted and organized by themes focusing on perceptions, practices, preferred care, needs, barriers and role of main care givers regarding continuum of care of pregnant women. Content analysis will be performed; and contrasts and comparisons will be made.

\section{Second Phase: Quantitative data analysis for explanatory design}

SPSS version 16 and STATA version 15 will be used for analysis. Because of adopting a quasi-experimental design, propensity score will be used for matching of respondents to create a valid and counterfactual comparison group and to control confounding. Descriptive statistics will be shown by frequency and percentage for categorical variables and continuous variables will be summarized by mean and standard deviation. For post-intervention data analysis: awareness on CoC and outcomes variables: ANC visits, birth by skilled attendance and post-natal checks between intervention and comparison groups will be analyzed by $x^{2}$ test. For effect of intervention: multiple logistic regression analysis will be applied to compare completion of CoC between two groups by adjusting possible confounders such as awareness of main care givers, education of pregnant women, frequency of ANC visits for current pregnancy and source of health information. The results of the model will be presented using adjusted odds ratios (OR) with $95 \%$ confidence interval (CI) and p value $<0.05$ will be set as level of statistically significance.

\section{Third Phase: Qualitative data analysis}

End line qualitative findings from IDIs will also be started from data collection to until it ended. The interviews will be transcribed on the day of data collection by listening audio records repeatedly. Initial analysis will be started by pre-categorizing themes. After that, main themes such as perceptions of main care-givers on continuum of care for pregnant women, their preferred methods, problems and difficulties will be explored by performing content analysis.

\section{III.RESULTS}

The proposal of this study was submitted to the board of studies and ethical approval has been received from the Institutional Review Board (IRB) of University of Public Health, Yangon. It is also registered by Preliminary Registration: PLRID-00568_V3 at Myanmar Health Research Registry, Department of Medical Research, Yangon, The Republic of the Union of Myanmar. Focus group discussions were done within second and third week of February 2020 in Tike Kyi township. Three FGDs included 7 pregnant women, 7 main care-givers of pregnant women and 9 midwives in each session respectively. First 
qualitative data analysis is ongoing for development of intervention package. Pretesting among 20 pregnant women and their main care givers has been done and some areas of questionnaire are modified. After preparation of intervention package, midwives from intervention areas will be recruited and trained about health education for main care givers of pregnant women. Then baseline quantitative data collection will be conducted in both intervention and control areas. Follow-up data collections by both quantitative and qualitative are expected to be completed by June 2021. Final report will be submitted at the end of 2021.

\section{IV.DISCUSSION}

Being an intervention conducting among care-givers of pregnant women, results may be answered for some contradictions regarding the importance of men and families for maternal health according to previous studies [21,25,26,27,28]. The WHO IFC framework highlighted to conduct interventions to address the need for improving maternal health [22]. By conducting this study, we will have results about awareness on continuum of care among main care-givers, the completion of continuum of care among pregnant women and the perceptions on continuum of care among main care-givers. Therefore, this can point out the effect of health education intervention of main care-givers on completion of continuum of care. Better understanding of the gaps in seeking care and factors contributing to the gaps can be explored for successful program implementation. Moreover, the developed health education package is hoped to be disseminated to Township Medical Officer (TMO) and Basic Health Staffs (BHS) from control township if there is significant improvement on continuum of care among pregnant women by the intervention after the study.

One of the strengths of the study is intervention at community level and rural setting. Mixed methods by exploratory sequential design is applied to be strengthen the results of the study. Intervention package is developed based on the discussion of three groups with different experiences on continuum of care for maternal health. For main care-givers, health education is given by trained midwives who are conducting health services in the study areas and already familiar to antenatal, intra-natal and postnatal care of pregnant women. After intervention, not only continuum of care but also awareness and perception of main care givers will be explored. Data analysis is planned to reduce the effect of confounders by matching and multiple logistic regression. Moreover, this is the first interventional study among main care givers of pregnant women regarding continuum of care for maternal health.

There are some limitations of the study is that health education package is not reached to the control clustere. For awareness on continuum of care among main care givers, only post intervention assessment by assuming same background is done without preliminary survey. Health education is given only one time for main care givers of pregnant women from intervention cluster and therefore it is not ascertained to be memorized for long term. The continuous usage of pamphlets distributed as tool for health messages is not sure for through out of antenatal to postnatal periods of pregnant women.

\section{V.CONCLUSION}

By developing intervention package for health education among main care-givers, important factors for maternal health can be reached within close-groups who can give supports for pregnant women. For the continuum of care among pregnant women, needs and gaps to be fulfilled will be explored by this study and may be useful for further researches. Moreover, the results will help to determine whether it is applicable for current activities for maternal health promotion in Myanmar.

\section{ACKNOWLEDGEMENT}

We would like to express our heartfelt thanks to members of Institutional Review Board (IRB) of University of Public Health, Yangon and all involved health care personnel, local authorities, study participants from respected areas. 


\section{REFERENCES}

1. The maternal, newborn, and child health continuum of care. [Cited 2019 Sept 12]. Available from: https:// www. who.int/ pmnch/media/ publications/aonsection II.pdf.

2. Kerber K, Graft-Johnson Jd, Bhutta ZA, Okong P, Starrs A and Lawn JE. Continuum of care for maternal, newborn, and child health: From slogan to service delivery. Lancet 2011; 370 (9595): 1358-1369.

3. Antenatal Care Chapter 2, Section 3. [Cited 2019 Sept 12]. Available from: https:// www. who.int/ pmnch/ media/ publications/ aonsectionIII.pdf .

4.Kikuchi K, Ansah EK, Okawa S, Enuameh Y, Yasuoka J, Nanishi K, Shibanuma A, et al. Effective Linkages of Continuum of Care for Improving Neonatal, Perinatal, and Maternal Mortality: A Systematic Review and Meta-Analysis. PLoS ONE 2015; 10(9): e0139288. doi:10.1371 /journal. pone. 0139288.

5.World Health Organization recommendations on antenatal care for a positive pregnancy experience: Summary. Highlights and Key Messages from World Health Organization's 2016 Global Recommendations for Routine Antenatal Care. [Cited 2019 Aug 16]. Available from: www.mcsprogram.org.

6.Bernis Ld, Sherratt DR, Zahr CA and Lerberghe WV. Skilled attendants for pregnancy, childbirth and postnatal care. Br Med Bull 2003; 67(1): 39-57. doi: 10.1093/bmb/ldg017.

7.Amouzou1 A, Mehra V, Carvajal-Aguirre L, Khan SM, Sitrin D, Lara VL. Measuring postnatal care contacts for mothers and newborns: An analysis of data from the MICS and DHS surveys. JOGH. 2017;7(2):020502. doi:10.7189/jogh. 07. 020502.

8.Tiwari HC and Gupta SK. A study on postnatal care and its correlates among recently delivered women visiting to BRD medical college Gorakhpur. IJCMPH 2017;4(5):1548-1551. DOI: http:// dx. doi.org/10.18203/2394-6040.Ijcmph 20171761.

9. Trends in estimates of maternal mortality ratio (MMR; maternal deaths per 100,000 live births) 2000-2017. [Cited 2019 Sept 8]. Available from: https:// data.unicef.org/topic/ maternal-health/maternal-mortality.

10.Ministry of Health and Sports, Department of Public Health, Maternal and Reproductive Health Division. Strategy to End Preventable Maternal Mortality in Myanmar (2017-2021): Nay Pyi Taw, Myanmar; 2018, p.46-47.

11.Strategies towards ending preventable maternal mortality (EPMM). [Cited 2019 Sept 8]. Available from: http://www.Everywoman everychild.org/images/EPMM_final_report_2015.pdf.

12.Remarkable progress, new horizons and renewed commitment, Ending preventable maternal, newborn and child deaths in South-East Asia Region. [Cited 2019 Oct 8]. Available from: www.searo.who.int/ entity/ child_adolescent/topics /child_ health 19789290225294.pdf.

13.Kywe MM, Saw S, Thida M, Mya WW and Myint SS. Determinants of utilization of antenatal care services among women in Kungyangone Township, Myanmar. In: 45th Myanmar Health Research Congress, Yangon, Department of Medical Research; 2017.

14.Thandar MM, Kywe MM, Yi KH and Nyunt KK. Antenatal care pattern and maternal and perinatal outcomes. In: 45th Myanmar Health Research Congress, Yangon, Department of Medical Research; 2017.

15. Accessibility and utilization of postnatal care among rural mothers at primary health care level in Myanmar. [Cited 2019 Sept 8]. Available from: https://www.3mdg. org/en/ publication / accessibility-and-utilization-of-post-natal-care-among-rural-mothersat-primary-health.

16.Kabir R, Haider MR and Kordowicz M. A Cross-sectional study to explore the challenges faced by Myanmar women in accessing antenatal care services. ebph 2018;15(3): e12933-1- e12933-7. DOI: 10.2427/12933.

17.Min MS, Maw SYT, Oo KWW, Whelan R, Myint ZT, Thura S et al. (Postnatal care services utilization and associated factors among mothers in hard-to-reach areas in Kayin State, Myanmar. In: 47th Myanmar Health Research Congress. Yangon, Department of Medical Research; 2019. 
18.Eraky EM. (2018) Determinants of Non- Adherence to Antenatal Care Among Pregnant Women. IOSR-JNHS 2018;17(3):1524.

19. Mon AS, Phyu MK, Thinkhamrop W and Thinkhamrop B. Utilization of full postnatal care services among rural Myanmar women and its determinants: a cross-sectional study. F1000Research 2018; 7(1167).

20.World Health Organization recommendations on health promotion interventions for maternal and newborn health. [Cited 2019 Aug 16]. Available from: https:// apps. who. Int /iris /bitstream/ handle/10665/ 172427/9789241508742_report_eng.pdf.

21.Mullany BC, Becker S and Hindin MJ. The impact of including husbands in antenatal health education services on maternal health practices in urban Nepal: results from a randomized controlled trial. Health education research 2007; 22(2): 166-176. doi:10. 1093/her/cyl060.

22.WHO, Department of Making Pregnancy Safer. Geneva. Working with individuals, families and communities to improve maternal and newborn health. Geneva: WHO Press; 2010, p.3-26.

23.Ministry of Health and Sports (MOHS) and ICF. Myanmar Demographic and Health Survey 2015-16. Nay Pyi Taw, Myanmar and Rockville, Maryland USA.MOHS and ICF; 2017, p.121.

24. Ministry of Health and Sports. MCH Handbook. Nay Pyi Taw: Myanmar MOHS; 2019.

25.Sood S, Chandra U, Palmer A and Molyneux I. Measuring the effects of the SIAGA behaviour change campaign in Indonesia with population-based survey results. Baltimore, Maryland: JHPIEGO;2004. p.15-46.

26.Purdin S, Khan Tand Saucier R. Reducing maternal mortality among Afghan refugees in Pakistan. Int J Gynaecol Obstet; 2009:105(1): 82-85.

27.Turan JM, Tesfagiorghis M, Polan ML. Evaluation of a Community Intervention for Promotion of Safe Motherhood in Eritrea. J Midwifery Womens Health 2011; 56(1): 8-17. doi: 10.1111/j.1542-2011.2010.0000 .x.

28.Rahman AE, Perkins J, Mazumder T, Haider MR, Siddique AB, Capello C et al. Capacities of women and men to improve maternal and newborn health: Effect of a community-based intervention package in rural Bangladesh. J Glob Health 2019; 9(1): 010413 doi: $10.7189 /$ jogh. 09.010413 .

CONFLICT OF INTEREST: No conflicts of interest

Corresponding Author: May Soe Aung, M.B.,B.S, MPH, Ph.D (Public Health) Candidate, University of Public Health, Yangon, Myanmar, Email: maysoeag2010@gmail.com 